\title{
Asociación de leptina con factores cardiometabólicos en escolares y adolescentes con hiperplasia suprarrenal congénita
}

\author{
Jessie Nallely Zurita-Cruz, ${ }^{1}$ Miguel Ángel Villasís-Keever, ${ }^{2}$ Leticia Damasio-Santana, ${ }^{3}$ \\ Leticia Manuel-Apolinar, ${ }^{3}$ Rosalba Ferrusca-Ceja, ${ }^{4}$ Elisa Nishimura-Meguro, ${ }^{4}$ \\ Aleida de J. Rivera-Hernández ${ }^{4}$ y Eulalia Garrido-Magaña ${ }^{4}$ \\ 'Unidad de Investigación en Nutrición Médica; ${ }^{2}$ Unidad de Investigación Médica en Epidemiología Clínica; ${ }^{3}$ Unidad de Investigación Médica en \\ Enfermedades Endocrinas, Diabetes y Metabolismo; ${ }^{4}$ Hospital de Pediatría, Departamento de Endocrinología Pediátrica. ${ }^{1-4}$ Instituto Mexicano del \\ Seguro Social, Centro Médico Nacional Siglo XXI. Ciudad de México, México
}

\section{Resumen}

Introducción: En la hiperplasia suprarrenal congénita (HSC), la obesidad, la hiperinsulinemia y los niveles de leptina se encuentran incrementados. Objetivo: Identificar la frecuencia de los factores de riesgo cardiometabólico (FRC) en niños y adolescentes con HSC y explorar la relación con los niveles de leptina. Método: Estudio transversal de 40 pacientes a quienes se realizó somatometría y evaluación de glucosa, insulina, triglicéridos, 17-hidroxiprogesterona, leptina, colesterol HDL y $L D L$ en ayuno. Los pacientes fueron clasificados por el número de FRC y se analizaron los niveles de leptina con Kruskal-WaIlis. Se aplicó correlación de Pearson entre la leptina, puntuación Z del índice de masa corporal (zIMC) y porcentaje de grasa corporal. Resultados: 50 \% de los pacientes presentó obesidad y sobrepeso, 59 \% hipertrigliceridemia, $40 \%$ hipoalfalipoproteinemia, $27.5 \%$ colesterol LDL alto y $22.5 \%$ resistencia a la insulina. Hubo correlación positiva entre leptina y porcentaje de grasa corporal $(r=0.64)$, el zIMC $(r=0.55)$ y el número de FRC $(r=0.65)$. En el análisis multivariado ajustado por obesidad, los niveles de leptina se asociaron con el número de FRC. Conclusión: La HSC tuvo alta frecuencia de FRC y al parecer la leptina se asoció con perfil cardiometabólico más adverso en sujetos con obesidad y sobrepeso.

PALABRAS CLAVE: Hiperplasia suprarrenal congénita. Leptina. Pediatría.

\begin{abstract}
Introduction: In congenital adrenal hyperplasia (CAH), obesity, hyperinsulinemia and leptin levels are increased. Objective: To identify the frequency of cardiometabolic risk factors (CRF) in children and adolescents with CAH and to explore the relationship with leptin levels. Method: Cross-sectional study of 40 patients who underwent anthropometric measurements and had fasting glucose, insulin, triglycerides, 17-hidroxyprogesterone, leptin, HDL and LDL-cholesterol assessed. The patients were classified according to the number of CRFs, and leptin levels were analyzed with the Kruskal-Wallis test. Pearson's correlation was applied between leptin, body mass index (BMI) z-score and body fat percentage. Results: Fifty percent of the patients had obesity and overweight, 59\% had hypertriglyceridemia, 40\%, hypoalphalipoproteinemia, 27.5\%, high LDL-cholesterol and $22.5 \%$ insulin resistance. There was positive correlation between leptin and body fat percentage ( $r=0.64), B M I z-s c o r e(r=0.55)$ and the number of CRFs ( $r=0.65)$. In the obesity-adjusted multivariate analysis, leptin levels were associated with the number of CRFs. Conclusion: CAH had a high frequency of CRFs and leptin appeared to be associated with a more adverse cardiometabolic profile in subjects with obesity and overweight.
\end{abstract}

KEY WORDS: Congenital adrenal hyperplasia. Leptin. Pediatrics.

Fecha de recepción: 06-05-2017

Fecha de aceptación: 18-05-2017

DOI://dx.doi.org/10.24875/GMM.18002952
Gac Med Mex. 2018;154:202-208

Disponible en PubMed www.gacetamedicademexico.com 


\section{Introducción}

La hiperplasia suprarrenal congénita (HSC) es un trastorno autosómico recesivo caracterizado por incremento en la producción de andrógenos y deficiencia de cortisol. ${ }^{1}$ El objetivo del tratamiento es dar una adecuada sustitución de cortisol para prevenir crisis adrenales y suprimir la producción excesiva de andrógenos. ${ }^{2}$ Sin embargo, para lograr este efecto se deben administrar dosis suprafisiológicas de cortisol, con la que la línea entre la supresión del hiperandrogenismo endógeno y provocar síndrome de Cushing iatrogénico es muy delgada. ${ }^{3}$

Estudios en adultos con HSC han demostrado alta frecuencia de obesidad, ${ }^{4}$ hipercolesterolemia, resistencia a la insulina $(\mathrm{RI})^{5}$ e hipertensión arterial; ${ }^{6}$ las dosis suprafisiológicas de cortisol puede ser un posible detonante en el desarrollo de factores de riesgo cardiometabólico (FRC). ${ }^{7}$

Por otro lado, Völkl et al. describieron a 89 niños y adolescentes alemanes con HSC en quienes se identificó obesidad en $16.8 \%$, proporción superior en comparación con la reportada en niños y adolescentes de la población general alemana $(2.27 \%) .{ }^{8}$ En un estudio que incluyó a 22 pacientes con edad entre siete y 22 años se observaron valores más altos en la puntuación Z del IMC (ZIMC) que en la población sana. ${ }^{9}$

La leptina es una hormona peptídica ${ }^{10}$ producida por los adipocitos, regulada por neuropéptidos hipotalámicos ${ }^{11}$ e implicada en el metabolismo energético, la termogénesis y la ganancia de peso. ${ }^{12}$ En los seres humanos, la leptina se ha asociado con la obesidad y es considerada un factor de riesgo para el síndrome metabólico. ${ }^{13}$ La leptina desempeña un papel permisivo en el desarrollo puberal. Algunos estudios relacionan el inicio de la pubertad con el aumento de los niveles de leptina. ${ }^{14}$ Estas acciones pueden explicar por qué los niveles de leptina son diferentes según la edad, el sexo, la grasa corporal y la etapa de la pubertad. Tanto la obesidad y la pubertad pueden estar asociadas con aumento de la leptina. ${ }^{15}$

Particularmente en los pacientes con HSC, las concentraciones séricas altas de andrógenos alteran el metabolismo de la leptina, provocando hiperleptinemia; por otro lado, los receptores beta adrenérgicos, que regulan el metabolismo de los lípidos y la termogénesis, se encuentran deficientes en los pacientes con HSC..$^{16}$ Lo anterior condiciona un ambiente adverso, provocando aumento en el desarrollo de los FRC.
Se ha descrito que adultos y adolescentes con HSC presentan mayor frecuencia de $\mathrm{FRC}$, con asociación entre los niveles de leptina y algunos FRC. Hasta el momento no se ha estudiado si los FRC y la leptina están elevados desde la etapa prepuberal, así como la relación entre el número de FRC y los niveles de leptina.

El objetivo de este estudio fue determinar la frecuencia de los FRC en escolares y adolescentes con HSC y su relación con los niveles de leptina.

\section{Método}

Estudio transversal de niños y adolescentes mexicanos con HSC, que se llevó a cabo entre diciembre de 2014 y julio de 2015. Se incluyeron pacientes con edades que oscilaron entre seis a 16 años, quienes se encontraban en seguimiento al menos 12 meses por endocrinología pediátrica y con sustitución hormonal con base en esteroides sistémicos. Se seleccionaron 46 pacientes que cumplieron con los criterios de inclusión, se excluyeron seis: dos no tenían exámenes completos y en cuatro, los padres negaron el permiso para que sus hijos participaran en el estudio. El protocolo de investigación fue aprobado por el comité de investigación del hospital con el número R-2014-3603-32. Los padres firmaron la carta de consentimiento informado y los pacientes mayores de 8 años firmaron la carta de asentimiento, de acuerdo con las recomendaciones de la Declaración de Helsinki.

\section{Evaluación clínica y antropométrica}

Las variable somatométricas registradas fueron peso, estatura, circunferencia de cintura y porcentaje de grasa corporal (\%GC) medido por el método de bioimpedancia (Tanita BC-568); estas mediciones fueron realizadas por un nutricionista certificado. Mediante exploración física se identificó el estadio puberal de acuerdo con la escala de Tanner. La presión arterial fue el promedio de tres mediciones tomadas con un esfigmomanómetro en distintas ocasiones. La RI se estimó utilizando el modelo de homeostasis (HOMA):17

$$
\begin{aligned}
R I= & \text { insulina (microunidades por mililitro) } x \\
& \text { glucosa (milimolas por litro/22.5) }
\end{aligned}
$$

Los FRC analizados fueron obesidad (IMC de percentil $\geq 95$ ), hipertensión arterial sistémica (presión sistólica $\geq$ percentil 90 o presión diastólica $\geq$ percentil 90 para la edad), ${ }^{18}$ alteración de la glucosa en 
ayuno (glucosa plasmática $\geq 100 \mathrm{mg} / \mathrm{dL}$ ), ${ }^{19}$ hipertrigliceridemia (triglicéridos [TGL] plasmáticos $\geq$ percentil 90 de acuerdo con la edad y el sexo), hipoalfalipoproteinemia (colesterol de alta densidad $[\mathrm{HDLC}]<$ percentil 10 para edad y sexo, ${ }^{20}$ colesterol de baja densidad (LDLC) $>100 \mathrm{mg} / \mathrm{dL}$ y RI (puntuación HOMA > 3.4). ${ }^{21}$ El porcentaje de grasa fue considerando anormal de acuerdo con el sexo: en mujeres cuando era $>30 \%$ y en varones $>20 \%{ }^{22,23}$

La dosis de glucocorticoides se expresó como la dosis acumulada por la superficie corporal (sc) al día (miligramos por metro cuadrado [mg/ $\left./ \mathrm{m}^{2} \mathrm{sc}\right]$ ), tomando el promedio de la dosis de los últimos seis meses antes del estudio. Todas las dosis de glucocorticoides se convirtieron en dosis de hidrocortisona $(\mathrm{HC}): 1 \mathrm{mg}$ de prednisona $=4 \mathrm{mg}$ de $\mathrm{HC}$ y $1 \mathrm{mg}$ de prednisolona = $5 \mathrm{mg}$ de HC. Se consideró que la HSC estaba con adecuado control cuando 17-hidroxiprogesterona (17OHP) fue $<10 \mathrm{ng} / \mathrm{mL}$.

\section{Evaluación bioquímica}

Se obtuvieron sueros de muestras de sangre por venopunción, previo ayuno de 12 horas. La glucosa, TGL, LDLc, HDLc se determinaron por método enzimáticos colorimétricos (Bayer Diagnostics, Puteaux, Francia). La insulina se midió por quimioluminiscencia (Roche-Hitachi Modular P y D), 17-OHP y leptina (Human Leptin Duo Set, DY 398®) se midieron con ensayo de inmunoabsorción ligado a enzimas (ELISA) (R \& D Systems, Minneapolis, MN, EE. UU.). Todos los análisis de ELISA se determinaron en Instrumentos Multiskan EX, MTX® (Lab Systems Inc.), por duplicado según lo recomendado por el fabricante. El coeficiente de variación intra e interensayo para todas las mediciones fue $<7 \%$. También se incluyó una curva de estandarización para cada ensayo.

\section{Análisis estadístico}

Los datos se analizaron utilizando el programa estadístico SPSS versión 17.0. En virtud de que no se encontró distribución normal para las variables cuantitativas, se calculó mediana y valores mínimo y máximo.

Los pacientes fueron clasificados según el número de FRC y se compararon los niveles de leptina con la prueba de Kruskal-Wallis. Mediante el coeficiente de Pearson se determinó la correlación entre leptina y zIMC y \%GC. Se realizaron diversos modelos de regresión lineal para identificar las variables asociadas con la predicción del número de $\mathrm{FRC}$, en los que se incluyeron leptina, 17OHP, dosis de HC, ausencia o presencia de la pubertad, zIMC y el \%GC.

\section{Resultados}

Se analizaron 40 pacientes (26 mujeres), con edades entre seis y 16 años (mediana de 11.9 años). La relación de la edad ósea/edad cronológica fue mayor a uno en la mayoría de los casos, debido a una edad ósea adelantada en estos pacientes. La variedad de HSC más frecuente fue la perdedora de sal, seguida por la virilizante simple. Todos los pacientes recibían tratamiento de reemplazo con glucocorticoides, con una mediana de la dosis $\mathrm{HC}$ de $15.7 \mathrm{mg} / \mathrm{m}^{2} \mathrm{sc}$ (Tabla 1).

Respecto a los FRC, 14 (35\%) y ocho (20\%) sujetos tenían obesidad y sobrepeso, respectivamente; en $20(59 \%)$ se identificó hipertrigliceridemia, $16(40 \%)$ tuvieron hipoalfalipoproteinemia, 11 (27.5\%) aumento del LDLc, nueve (22.5\%) RI, tres (7.5\%)

Tabla 1. Datos generales de 40 pacientes pediátricos con hiperplasia suprarrenal congénita

\begin{tabular}{|c|c|c|}
\hline & $\mathrm{n}$ & $\%$ \\
\hline \multicolumn{3}{|l|}{ Sexo } \\
\hline Femenino & 26 & 65 \\
\hline Masculino & 14 & 35 \\
\hline \multicolumn{3}{|l|}{ Clasificación de la HSC } \\
\hline Perdedora de sal & 30 & 75 \\
\hline Virilizante simple & 2 & 5 \\
\hline Tardía & 8 & 20 \\
\hline \multicolumn{3}{|l|}{ Terapia con glucocorticoides } \\
\hline Dexametasona & 7 & 17.5 \\
\hline Prednisolona & 9 & 22.5 \\
\hline Prednisona & 23 & 56.5 \\
\hline Hidrocortisona & 1 & 2.5 \\
\hline Adecuado control de la HSC & 16 & 40 \\
\hline \multicolumn{3}{|l|}{ Estadio de Tanner } \\
\hline I & 9 & 25 \\
\hline II & 8 & 22.2 \\
\hline III & 4 & 11.1 \\
\hline IV & 10 & 27.8 \\
\hline \multirow[t]{2}{*}{ V } & 5 & 13.9 \\
\hline & Mediana & Mínimo-máximo \\
\hline Edad cronológica (años) & 12 & $6-16$ \\
\hline Edad ósea (años) & 11 & $6-16$ \\
\hline 17-hidroxiprogesterona (ng/mL) & 11.11 & $0.20-444.7$ \\
\hline Dosis de glucocorticoides (mg/m² sc) & 15.7 & 4.9-33.1 \\
\hline
\end{tabular}


hipertensión arterial sistémica y uno con glucosa en ayuno alterada. De los 40 sujetos, 33 (77.5\%) tenían uno o más FRC; en concreto, $25 \%$ tenía un factor, $20 \%$ dos, $17.5 \%$ tres, $7.5 \%$ cuatro y $7.5 \%$ cinco. El \%GC se encontró por arriba de lo normal en $65 \%$ $(\mathrm{n}=26)$ de los pacientes (Tabla 2).

En total, solo $25 \%(\mathrm{n}=10)$ de los pacientes no presentaba FRC, $17.5 \%(n=7)$ un factor y $57.5 \%$ $(n=23)$ más de dos. Se comparó el número de FRC entre los sujetos con y sin obesidad: $92.8 \%(n=13)$ de los pacientes con obesidad presentaban dos o más FRC; de los pacientes sin obesidad, solo $38.46 \%$ $(n=10)$ presentaba dos o más, con significación estadística $(p=0.013)$.

Para identificar si los pacientes desde etapa prepuberal presentaban FRC y su frecuencia, se realizó un subanálisis de 14 sujetos con edades entre los seis y nueve años, diez (71.4\%) tenían sobrepeso y obesidad $(28.5 \%$ de sobrepeso y $42.8 \%$ obesidad), siete (50\%) hipertrigliceridemia, seis (42.8\%) hipoalfalipoproteinemia, tres $(21.4 \%)$ aumento del LDLc y tres (21.4\%) Rl; 12 (85.7\%) tenían al menos un FRC.

Hubo correlación positiva entre los niveles de leptina, \%GC ( $r=0.64)$ y zIMC ( $r=0.55)$ (Figura 1$)$. También se compararon y correlacionaron los niveles de

Tabla 2. Datos somatométricos y parámetros bioquímicos en 40 pacientes con hiperplasia suprarrenal congénita

\begin{tabular}{lcc}
\hline & Mediana & Mínimo-máximo \\
\hline Puntuación Z del IMC & 0.81 & $-1.62-3.26$ \\
Percentil del zIMC & 82 & $5-99$ \\
Perímetro de cintura (cm & 68 & $49-108$ \\
Percentil del perímetro de cintura & 50 & $10-90$ \\
Porcentaje de grasa corporal & 30.75 & $18.3-48.8$ \\
Femenino & 31.7 & $21.5-48.8$ \\
Masculino & 26 & $18.3-45$ \\
Glucosa en ayuno (mg/dL) & 85.15 & $66.6-109$ \\
LDLc (mg/dL) & 82.7 & $11-143$ \\
HDLc (mg/dL) & 53.2 & $26-77$ \\
Triglicéridos (mg/dL) & 94 & $32-204$ \\
Presión sistólica (mm Hg) & 100 & $70-135$ \\
Percentil de la presión sistólica & 67 & $50-80$ \\
Presión diastólica (mm Hg) & 60 & $50-70$ \\
Percentil de la presión diastólica & 50 & $50-90$ \\
Insulina en ayuno ( $\mu U \mathrm{UI} / \mathrm{mL}$ ) & 9.85 & $1.02-37.2$ \\
HOMA & 2.01 & $0.21-9.09$ \\
\hline IMC, índice de masa corporal; zlMC, puntuación Z del índice de masa corporal; LDLc, \\
colesterol de baja densidad; HDLc, colesterol de alta densidad
\end{tabular}

leptina con el número de $\mathrm{FRC}$, donde a mayor número de factores, mayor nivel de leptina (Figura 2).

Se realizó un modelo de regresión lineal para identificar las características de los pacientes que se podían relacionar con el número de FRC; se incluyó leptina, zIMC, \%GC, niveles de 17OHP y la dosis de HC. En el modelo se observó que la leptina y la 170HP se asociaron significativamente con el número de FRC. Posteriormente se hizo un modelo ajustado por estadio de pubertad y presencia de sobrepeso y obesidad, donde los niveles de leptina se relacionaron en forma independiente con el número de FRC (Tabla 3).

\section{Discusión}

En esta investigación, los pacientes con HSC tuvieron alta frecuencia de $\mathrm{FRC}$, tanto en etapa prepuberal como puberal. Asimismo, se determinó que los niveles de leptina se incrementan de acuerdo con el número de FRC: Particularmente, en los pacientes con sobrepeso y obesidad, los niveles de leptina se asociaron con un perfil cardiometabólico adverso.

\section{Comparación con otros estudios}

En este estudio, los pacientes incluidos mostraron alta frecuencia de sobrepeso y obesidad (55\%) en comparación con lo esperado para la población pediátrica normal en México (34.4 \%). ${ }^{24}$ Se comparó la frecuencia de los FRC con la reportada por Arlt et al., ${ }^{6}$ quienes describen sujetos adultos, de los cuales aquellos con HSC tuvieron mayor frecuencia de hipoalfalipoproteinemia (40 versus $14 \%$ ), menor frecuencia en el aumento del LDLc (27.5 versus $39 \%)$ y RI con frecuencia similar (22.5 versus $29 \%$ ), lo que llama la atención porque desde etapas pediátricas tempranas se observa alta frecuencia en la RI y presencia de dislipidemia.

La fisiopatología del desarrollo de FRC en los pacientes con HSC es múltiple, donde destaca las dosis suprafisiológicas de esteroides e hiperandrogenemia, ambos en forma crónica. No se sabe la edad en la cual los pacientes desarrollan los cambios incipientes clínicos y bioquímicos que determinan la presencia de estos factores cardiovasculares y metabólicos. Sin embargo, los resultados sugieren que desde los seis años ya existen manifestaciones.

La pubertad es una variable de confusión respecto a los FRC. ${ }^{25,26} \mathrm{El}$ aumento de la RI está relacionado con el inicio de la pubertad debido al aumento 

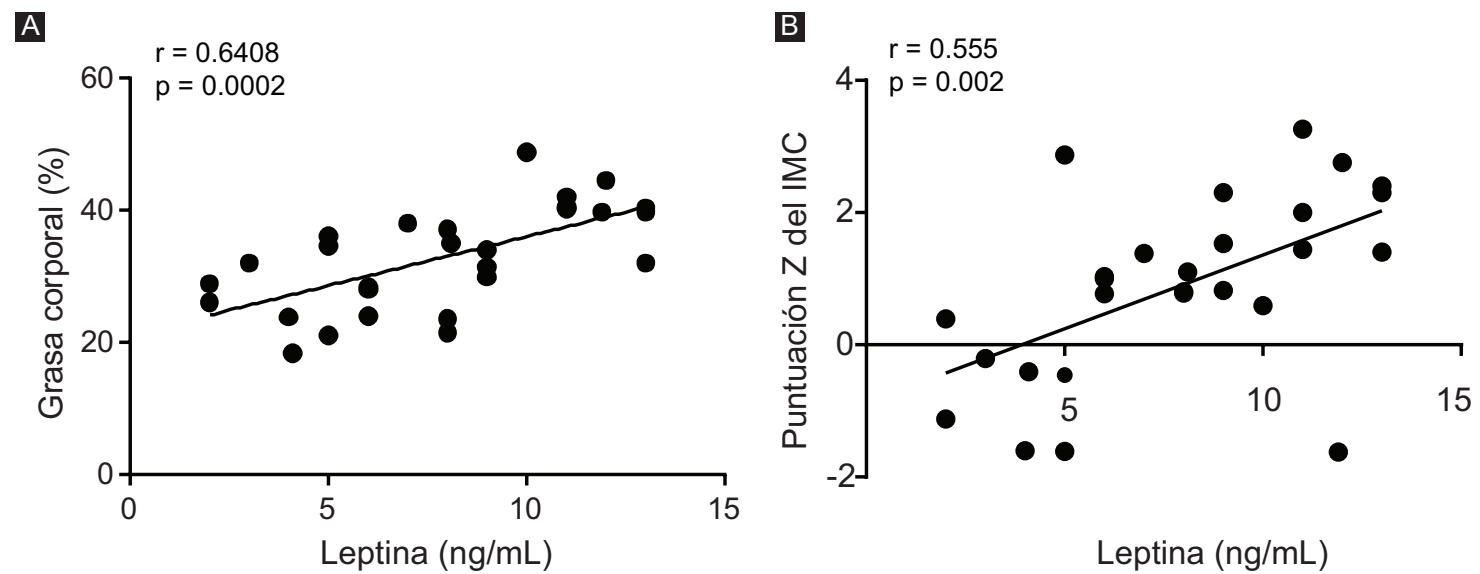

Figura 1. Correlación entre los niveles de leptina, porcentaje de grasa corporal (A) y puntuación Z del IMC (B) en pacientes con hiperplasia suprarrenal congénita.
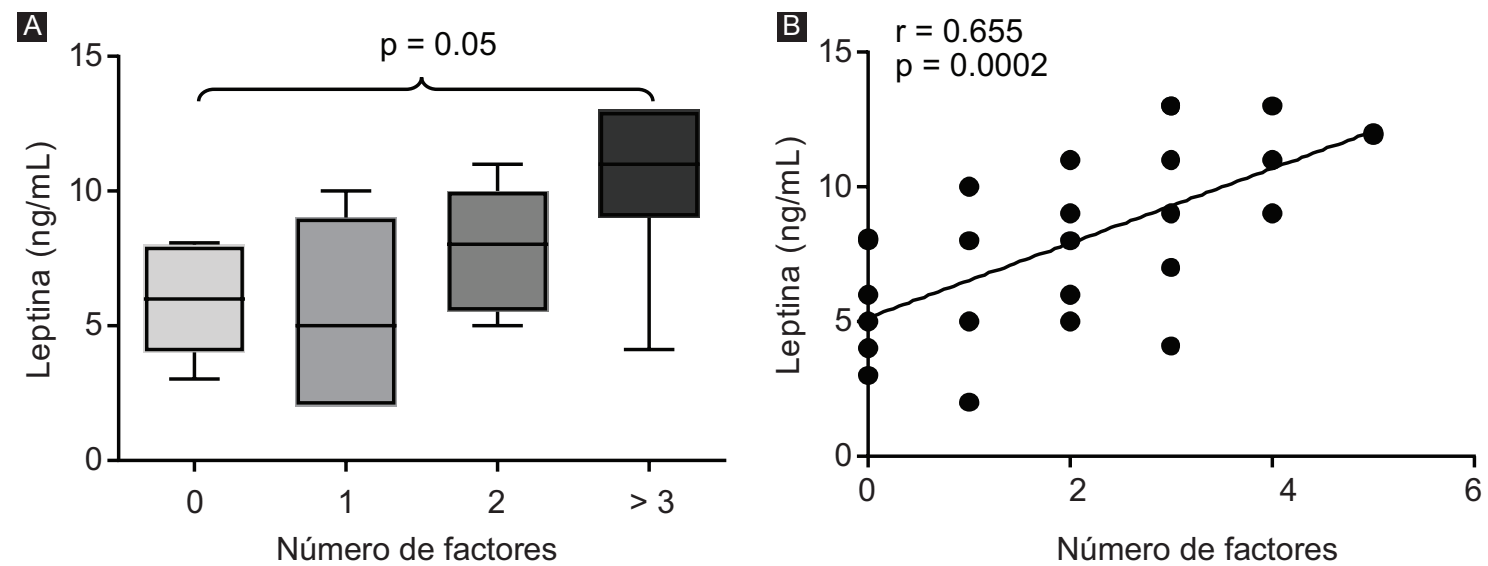

Figura 2. Comparación (A) y correlación (B) de los niveles de leptina y factores de riesgo cardiometabólico.

Tabla 3. Análisis de regresión lineal para predecir el número de factores de riesgo cardiometabólico

\begin{tabular}{|c|c|c|c|c|}
\hline & \multicolumn{2}{|c|}{ Modelo inicial } & \multicolumn{2}{|c|}{ Modelo ajustado } \\
\hline & $\beta^{*}$ & IC $95 \%$ & $\beta^{* *}$ & IC $95 \%$ \\
\hline Concentraciones séricas de leptina (ng/mL) & $0.269 \infty$ & $0.097-0.441$ & $0.322 \infty$ & 0.057-0.606 \\
\hline 17-hidroxiprogesterona (ng/mL) & $0.005 \infty$ & $0.0001-0.010$ & 0.004 & $-0.003-0.012$ \\
\hline Dosis de glucocorticoides (mg/m² sc) & -0.074 & $-0.166-0.011$ & 0.099 & $0.099-0.09$ \\
\hline Puntuación Z del IMC & -0.037 & $-0.451-0.376$ & -0.219 & $-0.861-0.423$ \\
\hline Grasa corporal (\%) & -0.003 & $-1.96-3.68$ & -0.014 & $-0.19-0.16$ \\
\hline
\end{tabular}

fisiológico de las hormonas contrarreguladoras necesarias para la maduración y crecimiento. ${ }^{27}$ Por esto se hizo un subanálisis de los FRC en los pacientes prepúberes, donde se observó que presentaban alta frecuencia de FRC, sin embargo, en el análisis multivariado no se demostró que la pubertad se asociara con aumento en los FRC; por lo tanto, la pubertad no fue una variable relacionada con los FRC.
Los mecanismos asociados con la obesidad (aumento del \%GC, ${ }^{16} \mathrm{RI}$ y elevación de la leptina) son diversos, particularmente en este grupo de sujetos. El exceso de andrógenos, como la dehidroepiandrosterona y la testosterona, pueden interferir en el metabolismo de la leptina a través de su señalización, provocando disminución en el receptor de OB de leptina, originando resistencia periférica $\mathrm{y}$, consecuentemente, 
hiperinsulinismo, resistencia a la insulina y obesidad, que altera la esteroidogénesis y aumenta la producción de andrógenos suprarrenales ${ }^{28-30}$ y ováricos. ${ }^{31}$

La obesidad y la HSC se asocian con altos niveles de leptina y deficiencia de los receptores beta adrenérgicos, que modulan el metabolismo de los lípidos y la termogénesis, lo que estimula el desarrollo de FRC. ${ }^{32,33}$ Lo anterior condiciona un ambiente proinflamatorio, aumento del riesgo del síndrome metabólico y ovario poliquístico, así como disminución en la eficacia del tratamiento con glucocorticoides. Esto explica la alta frecuencia de FRC identificados en los pacientes estudiados.

De acuerdo con las observaciones, desde la etapa escolar los pacientes con HSC requieren el inicio temprano de intervenciones dietético-ambientales y farmacológicas. Por ejemplo, el tratamiento farmacológico podría enfocarse a mejorar la $\mathrm{Rl}$ a través de sensibilizadores, como metformina, ${ }^{34}$ ya que en estos pacientes no hay forma de suspender el tratamiento con glucocorticoides ni modificar las fluctuaciones en los niveles de andrógenos, a pesar de estar controlados.

En este grupo de pacientes, que presentó alta frecuencia de obesidad, sobrepeso (55\%) y \%GC elevado (65\%), se observó que estas variables fueron confusoras respecto al número de FRC y niveles de leptina. Si bien, lo descrito es que la leptina se relaciona en forma directa con el \%GC y el zIMC, ${ }^{31}$ los pacientes con HSC es un grupo particular de sujetos en quienes la hiperandrogenemia ${ }^{28-32}$ altera el metabolismo, incluido el de la leptina, favoreciendo alteraciones cardiometabólicas. Esto se explica porque la leptina fue una variable independiente en el número de FRC en pacientes con HSC y obesidad. ${ }^{28}$

\section{Limitaciones del estudio}

El tamaño de la muestra fue pequeño y, por lo tanto, los resultados deben interpretarse como una tendencia y no como conclusiones. Asimismo, debido que es un estudio transversal, será conveniente llevar a cabo un análisis longitudinal a fin de identificar la edad en que inician los FRC. Las adipocitocinas, además de la leptina, y otros indicadores antropométricos deberán ser estudiados en futuras investigaciones.

En conclusión, se puede afirmar que los pacientes pediátricos con HSC tienen alta frecuencia de FRC y los niveles de leptina se asocian en forma independiente con un perfil cardiometabólico más adverso en el grupo de pacientes con obesidad.

\section{Bibliografía}

1. Speiser PW, White PC. Congenital adrenal hyperplasia. N Engl J Med. 2003:349:776-788.

2. Forest J, Maguelone $\mathrm{G}$. Recent advances in the diagnosis and management of congenital adrenal hyperplasia due to 21-hydroxylase deficiency. Hum Reprod Update 2004:10:469-485.

3. Speiser L, Azziz R, Baskin L, Ghizzoni L, Hensle T, Merke D, et al. Congenital adrenal hyperplasia due to steroid 21-hydroxylase deficiency: an endocrine society clinical practice guideline. J Clin Endocrinol Metab. 2010;95:4133-4160.

4. Kim J, Choi J, Kang E, Kim Y, Lee B, Yoo H. Long-term consequences of congenital adrenal hyperplasia due to classic 21-hydroxylase deficiency in adolescents and adults. Exp Clin Endocrinol Diabetes. 2017; $125: 196-201$

5. Sartorato P, Zulian E, Benedini S, Mariniello B, Schiavi F, Bilora F, et al. Cardiovascular risk factors and ultrasound evaluation of intima-media thickness at common carotids, carotid bulbs, and femoral and abdominal aorta arteries in patients with classic congenital adrenal hyperplasia due to 21-hydroxylase deficiency. J Clin Endocrinol Metab. 2007:92:1015-1018.

6. Arlt W, Willis D, Wild S, Krone N, Doherty E, Hahner S, et al. Health status of adults with congenital adrenal hyperplasia: a cohort study of 203 patients. J Clin Endocrinol Metab. 2010;95:5110-5121.

7. Falhammar H, Frisén L, Hirschberg A, Norrby C, Almqvist C, Nordenskjöld $A$, et al. Increased cardiovascular and metabolic morbidity in patients with 21-hydroxylase deficiency: a Swedish population-based national cohort study. J Clin Endocrinol Metab. 2015;100:3520-3528.

8. Völkl T, Simm D, Beier C, Dörr H. Obesity among children and adolescents with classic congenital adrenal hyperplasia due to 21-hydroxylase deficiency. Pediatrics. 2006;117:e98-e105.

9. Cornean R, Hindmarsh P, Brook C. Obesity in 21-hydroxylase deficient patients. Arch Dis Child. 1998;78:261-263.

10. Baptista C. Leptina. Acta Pediatr Port. 2002;15:281-285.

11. Moran O, Phillip M. Leptin: obesity, diabetes and other peripheral effects-a review. Pediatr Diabetes. 2003:4:101-109.

12. Steinberger J, Steffen L, Jacobs D, Moran A, Hong C, Sinaiko A. Relation of leptin to insulin resistance syndrome in children. Obesity Res. 2003:11:1124-1130.

13. Pilcová R, Sulcová J, Hill M, Bláha P, Lisá L. Leptin levels in obese children: effects of gender, weight reduction and androgens. Physiol Res. 2003:52:53-60.

14. Sanchez-Garrido M, Tena-Sempere M. Metabolic control of puberty: roles of leptin and kisspeptins. Horm Behav. 2013;64:187-194.

15. Garcia-Mayor R, Andrade M, Rios M, Lage M, Dieguez C, Casanueva F. Serum leptin levels in normal children: relationship to age, gender, body mass index, pituitary-gonadal hormones, and pubertal stage. J Clin Endocrinol Metab. 1997;82:2849-2855.

16. Charmandari E, Weise M, Bornstein S, Eisenhofer G, Keil M, Chrousos G et al.Children with classic congenital adrenal hyperplasia have elevated serum leptin concentrations and insulin resistance: potential clinical implications. J Clin Endocrinol Metab 2002;87:2114-2120.

17. Radziuk J. Insulin sensivity and its measurement: structural commonalities among the methodos. J Clin Endocrinol Metab 2000;85:4426-4433.

18. National High Blood Pressure Education Program Working Group on Hypertension Control in Children and Adolescents. Update on the 1987 Task Force Report on High Blood Pressure in Children and Adolescents: a working group report from the National High Blood Pressure Education Program. Pediatrics. 1996:98:649-658.

19. Zimmet P, Alberti G, Kaufman F, Tajima N, Silink M, Arslanian S, et al. The metabolic syndrome in children and adolescents. Lancet 2007;369:2059-2061.

20. de Ferranti S, Gauvreau K, Ludwig D, Neufeld E, Newburger J, Rifai N. Prevalence of the metabolic syndrome in American adolescents: findings from the Third National Health and Nutrition Examination Survey. Circulation. 2004;110:2494-2497.

21. García-Cuartero B, García-Lacalle C, Jiménez-Lobo C, González-Vergaz A, Calvo-Rey C, Alcázar-Villar M, et al. Indice HOMA y QUICKI, insulina y péptido $C$ en niños sanos. Puntos de corte de riesgo cardiovascular. An Pediatr. 2007;66:481-490.

22. Okasora K, Takaya R, Tokuda M, Fukunaga Y, Oguni T, Tanaka H, et al. Comparison of bioelectrical impedance analysis and dual energy X-ray absorptiometry for assessment of body composition in children. Pediatr Int. 1999;41(2):121-125.

23. Taylor R, Jones I, Williams S, Goulding A. Body fat percentages measured by dual-energy X-ray absorptiometry corresponding to recently recommended body mass index cutoffs for overweight and obesity in children and adolescents aged 3-18 y. Am J Clin Nutr. 2002; 76(6):1416-1421. 
24. Gutiérrez JP, Rivera-Dommarco J, Shamah-Levy T, Villalpando-Hernández S, Franco A, Cuevas-Nasu L, et al. Encuesta Nacional de Salud y Nutrición. México: Instituto Nacional de Salud Pública; 2012. p. 149-152. Disponible en: http://ensanut.insp.mx/informes/ENSANUT2012ResultadosNacionales.pdf

25. Caprio S, Tamborlane W. Effect of puberty on insulin action and secretion. Semin Reprod Endocrinol. 1994;12:90-96.

26. Travers S, Jeffers B, Bloch C, Hill J, Eckel R. Gender and tanner stage differences in body composition and insulin sensitivity in early pubertal children. J Clin Endocrinol Metab. 1995;80:172-178

27. Moran A, Jacobs D, Jr, Steinberger J, Hong C, Prineas R, Luepker R et al. Insulin resistance during puberty: results from clamp studies in 357 children. Diabetes. 1999;48:2039-2044.

28. L'Allemand D, Penhoat A, Lebrethon M, Ardevol R, Baehr V, Oelkers W, et al. Insulin-like growth factors enhance steroidogenic enzyme and corticotropin receptor messenger ribonucleic acid levels and corticotropin steroidogenic responsiveness in cultured human adrenocortical cells. $\mathrm{J}$ Clin Endocrinol Metab. 1996;81:3892-3897.
29. Biason-Lauber A, Zachmann M, Schoenle E. Effect of leptin on CYP17 enzymatic activities in human adrenal cells: new insight in the onset of adrenarche. Endocrinology. 2000;141:1446-1454.

30. Völkl T, Simm D, Körner A, Rascher W, Kiess W, Kratzsch J, et al. Does an altered leptin axis play a role in obesity among children and adolescents with classical congenital adrenal hyperplasia due to 21-hydroxylase deficiency? Eur J Endocrinol. 2009;160:239-247.

31. Hsueh W, Lyon C, Quinones M. Insulin resistance and the endothelium. Am J Med. 2004;117:109-117

32. Merke D, Chrousos G, Eisenhofer G, Weise M, Keil M, Rogol A, et al. Adrenomedullary dysplasia and hypofunction in patients with classic 21-hydroxylase deficiency. N Engl J Med. 2000;343:1362-1368.

33. Gonzaga N, Medeiros C, de Carvalho D, Alves J. Leptin and cardiometabolic risk factors in obese children and adolescents. J Paediatr Child Health. 2014;50(9):707-712.

34. Ibáñez L, Ong K, López-Bermejo A, Dunger D, de Zegher F. Hyperinsulinaemic androgen excess in adolescent girls. Nat Rev Endocrinol. 2014;10(8):499-508 